\title{
Endoscopic dissection of a symptomatic giant gastric leiomyoma arising from the muscularis propria
}

Gastrointestinal (GI) subepithelial masses represent a heterogeneous group of lesions, ranging from benign to malignant, for which management is sometimes challenging $[1,2]$. We report the case of an 85-year-old woman, with a history of coronary artery disease and chronic atrial fibrillation being treated with anticoagulant therapy, who underwent urgent upper $\mathrm{Gl}$ endoscopy for hemorrhagic shock and melena. During this procedure, a giant, $15-\mathrm{cm}$, non-pedunculated mass that was ulcerated on top was found at the greater curvature of the anterior wall of the stomach ( $\triangleright$ Fig. 1 ).

The patient then underwent radial endoscopic ultrasonography (EUS; GF-UE160AL5; Olympus), which showed a hypoechoic homogeneous intramural mass that was arising from the muscularis propria and was suspected to be a leiomyoma ( $\triangleright$ Fig. 2). In order to achieve a definitive diagnosis, EUS with fine needle aspiration (FNA) was performed (GF-UCT180; Olympus) using a 22-gauge needle (ExpectSlimLine; Boston Scientific). Histology and immunohistochemical staining revealed that the specimen was compatible with a leiomyoma (SMA positive, CD117 and CD34 negative). Total body computed tomography (CT) excluded metastatic disease.

Because this was a symptomatic hemorrhagic lesion and there was a need to continue anticoagulant therapy, an endoscopic dissection was performed. We used the HybridKnife T-type (ERBE Elektromedizin $\mathrm{GmbH}$ ) and a solution composed of $250 \mathrm{~mL}$ normal saline, $2 \mathrm{~mL}$ indigo carmine, and $1 \mathrm{~mL}$ epinephrine. The procedure took 115 minutes and resulted in an en bloc specimen, with no complications occurring ( $>$ Fig. 3 ; $>$ Video 1 ). However, because of its size, it was not possible to retrieve the whole lesion, which resulted in it being completely digested by gastric secretions by the following day ( $\triangleright$ Fig.4).

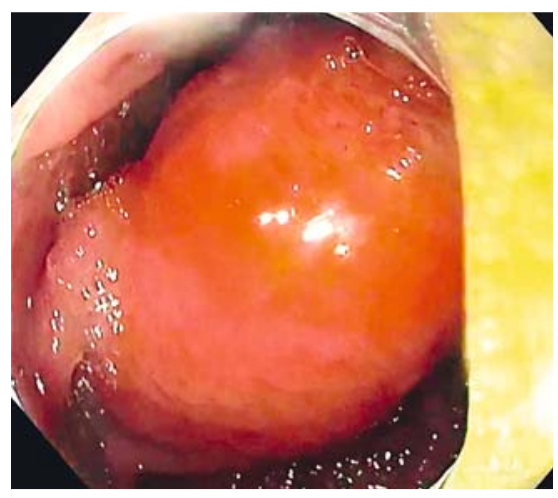

- Fig. 1 Endoscopic view showing the giant gastric subepithelial lesion.

Low molecular weight heparin was re-introduced 24 hours after the procedure and the patient was discharged 2 days later. Upper GI endoscopy and EUS performed 3 months later revealed a regular scar, without any remnant pathological tissue.

This case illustrates the feasibility and safety of endoscopic dissection of a symptomatic giant gastric leiomyoma, even in a high risk patient who was receiving ongoing anticoagulant therapy, in whom surgery would have carried considerable risk. Moreover, EUS-FNA achieved

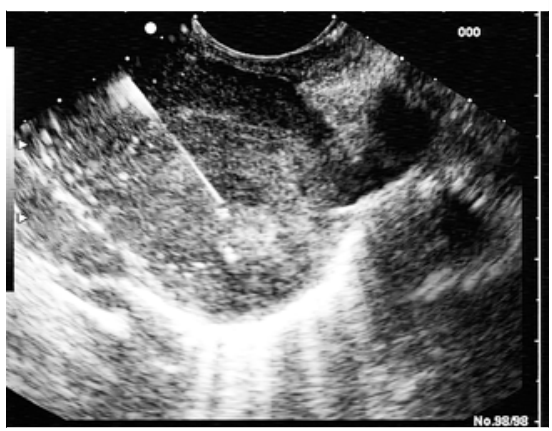

- Fig. 2 Endoscopic ultrasonography view of the lesion during performance of a fine needle aspiration.

an accurate evaluation of the lesion's layer of origin and its histopathologic characteristics, thereby allowing a definitive diagnosis to be made and the appropriate therapeutic option to be chosen.

Endoscopy_UCTN_Code_CCL_1AB_2AD_3AB

\section{Competing interests}

None

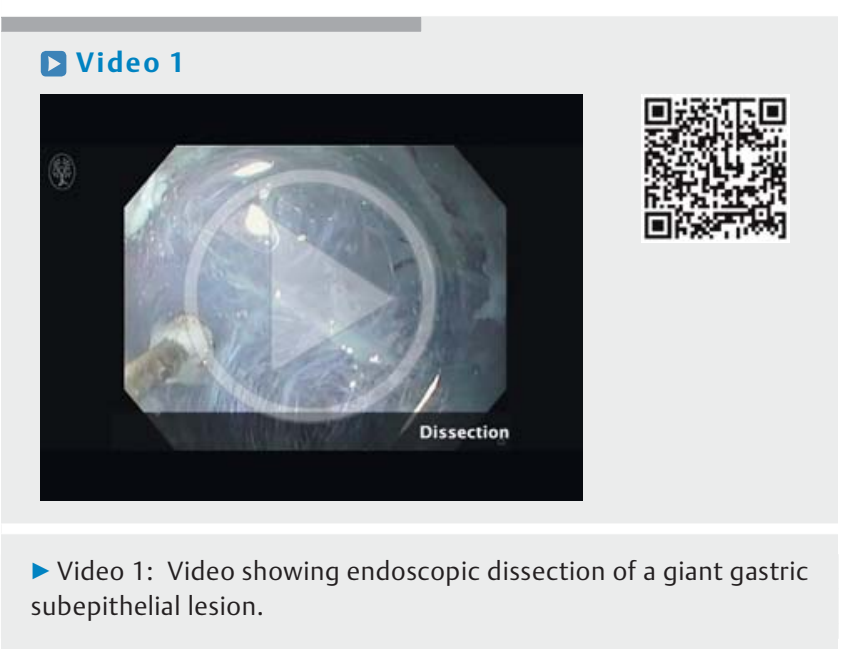




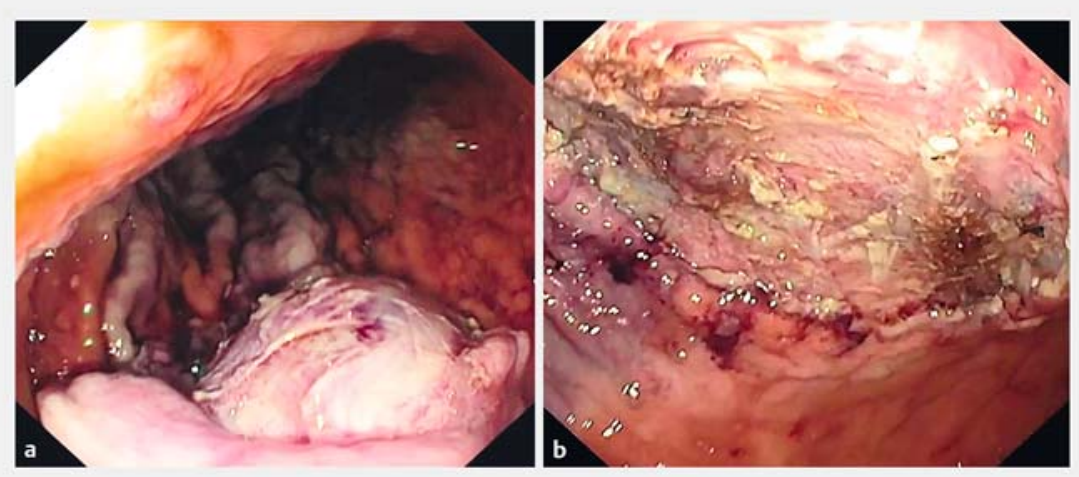

- Fig. 3 Endoscopic views showing: a the whole lesion after endoscopic dissection; $\mathbf{b}$ the appearance of the resection site immediately after endoscopic dissection.

The Authors

Mauro Manno ${ }^{1}$, Paola Soriani ${ }^{1}$, Vincenzo G. Mirante ${ }^{1}$, Giuseppe Grande ${ }^{2}$, Flavia Pigò ${ }^{2}$, Rita L. Conigliaro ${ }^{2}$

1 Digestive Endoscopy Unit, Azienda USL Modena, Ramazzini Hospital, Carpi, Italy

2 Rete Integrata Provinciale di Endoscopia Digestiva, Baggiovara Hospital, Modena, Italy
Corresponding author

\section{Paola Soriani, MD}

Digestive Endoscopy Unit, Azienda USL Modena - Ramazzini Hospital, Via Guido Molinari, 2, 41012 Carpi (MO), Italy Fax: +39-059-659500

paola.soriani@gmail.com

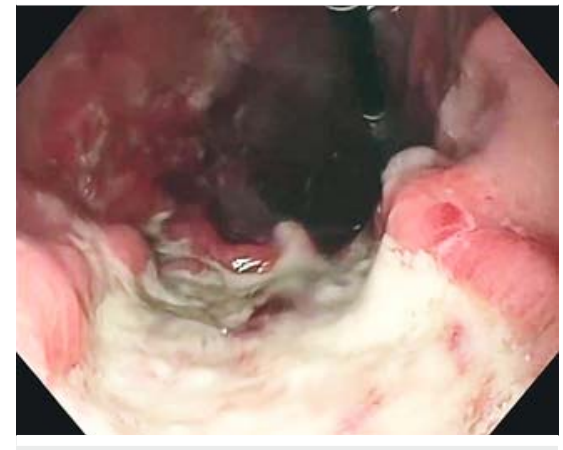

- Fig. 4 Endoscopic view of the resection site on the day following the procedure.

\section{References}

[1] He G, Wang J, Chen B et al. Feasibility of endoscopic submucosal dissection for upper gastrointestinal submucosal tumors treatment and value of endoscopic ultrasonography in pre-operation assess and post-operation follow-up: a prospective study of 224 cases in a single medical center. Surg Endosc 2016; 30: $4206-4213$

[2] Salah W, Faigel DO. When to puncture, when not to puncture: submucosal tumors. Endosc Ultrasound 2014; 3: $98-108$

\section{Bibliography}

DOI http://dx.doi.org/10.1055/s-0043-103402

Endoscopy 2017; 49: E141-E142

(c) Georg Thieme Verlag KG

Stuttgart · New York

ISSN 0013-726X 